\title{
Technique for Inferring Magnetic Helicity of Active Regions
}

\author{
Jongchul Chae \\ Astronomy Program, SEES, Seoul National University, Seoul 151-742, \\ Korea
}

Yong-Jae Moon

Korea Astronomy Observatory, Whaamdong, Yooseong-ku, Daejeon 305-348, Korea

\begin{abstract}
We provide the rationale for the wide applicability of the LCT method for inferring the magnetic helicity of an active region, with an illustrative application made to NOAA 10365 . We also report that the determined rate of helicity change is not much sensitive to the free parameters of the LCT method. The application of the method to active regions by several investigators has produced results suggesting that the magnetic helicity of a major active region with flux $10^{22} \mathrm{Mx}$ may be usually of the order of $10^{43} \mathrm{Mx}^{2}$ or less, being twisted at most 0.1 turns as a whole.
\end{abstract}

\section{Introduction}

It is now well known that the change rate of the magnetic helicity of a coronal volume $H$ is given by the equation (Berger \& Field 1984)

$$
d H / d t=\oint 2\left(\mathbf{B}_{t} \cdot \mathbf{A}_{p}\right) v_{n} d S-\oint 2\left(\mathbf{v}_{t} \cdot \mathbf{A}_{p}\right) B_{n} d S
$$

where the subscripts $t$ and $n$ refer to the components of magnetic field $\mathbf{B}$ and plasma velocity $\mathbf{v}$ that are tangential and normal to the surface, respectively. $\mathbf{A}_{p}$ is the vector potential of potential field. The method of local correlation tracking (LCT) was proposed by Chae (2001) as a way of tracing the horizontal motion of footpoints of predominantly vertical field lines and of determining the latter term in Eq. (1) - the so-called shearing term. Being successfully applied to the line-of-sight magnetograms taken by SOHO/MDI, this method has produced the meaningful estimates of magnetic helicity change in different active regions in relation to the prominence formation (Chae et al. 2001), flares (Moon et al. 2002a, b) and coronal mass ejections (Nindos et al. 2002, 2003). Subsequently generalizing the LCT method, Kusano et al. (2002) proposed a way of determining the other term in Eq. (1) - the advection term. They calculated the normal component of velocity from the induction equation and the LCT velocities. Very recently Demoulin \& Berger (2003) found that the amount of helicity change determined by Chae's LCT method, when applied to inclined magnetic fields, in fact includes the contribution of vertical motion, too, not to speak of that of horizontal motion, negating the need to add the extra 
advection term due to the vertical motion. This finding opens a possibility of the wider applicability of the LCT method than was originally proposed by Chae (2001).

\section{Rationale for the LCT Method}

The LCT technique determines the horizontal velocity from the lateral displacement of flux density pattern across the solar surface. It basically assumes that the velocity field is uniform locally in space and time so that it follows

$$
B_{n}(\mathbf{r}+\Delta \mathbf{r} / 2, t+\Delta t / 2) \approx B_{n}(\mathbf{r}-\Delta \mathbf{r} / 2, t-\Delta t / 2)
$$

for $\left|\mathbf{r}-\mathbf{r}_{0}\right|<\delta r$ and $\left|t-t_{0}\right|<\delta t$. The LCT velocity is defined as $\mathbf{v}_{\mathrm{LCT}}\left(\mathbf{r}_{0}, t_{0}\right)=$ $\Delta \mathbf{r} / \Delta t$. Note that for the LCT technique to work best the magnetic flux density should vary sharply in space with a length scale shorter than that of the velocity variation. Under this condition $\mathrm{Eq}(2)$ can be recast into

$$
\partial B_{n} / \partial t+\nabla_{t} \cdot\left[\mathbf{v}_{\mathrm{LCT}} B_{n}\right] \approx 0
$$

where the operator $\nabla_{t}$ refers to the tangential component of the gradient operator, which is defined on the photospheric surface.

On the other hand, one can start with the well-known magnetic induction equation and derive the equation

$$
\partial B_{n} / \partial t+\nabla_{t} \cdot\left[\mathbf{v}_{t} B_{n}-v_{n} \mathbf{B}_{t}\right]=\partial B_{n} / \partial t+\nabla_{t} \cdot\left[\mathbf{u} B_{n}\right]=0
$$

which looks very similar to $\mathrm{Eq}(3)$ with the newly introduced velocity $\mathbf{u}$ defined by $\mathbf{u} B_{n} \equiv \mathbf{v}_{t} B_{n}-v_{n} \mathbf{B}_{t}$. Note that $\mathbf{u}$ stands for the apparent motion of footpoints of field lines across the photospheric surface. The two kinds of velocity $\mathbf{u}$ and $\mathbf{v}_{\mathrm{LCT}}$ are related to each other via $\nabla_{t} \cdot\left(\mathbf{v}_{\mathrm{LCT}} B_{n}-\mathbf{u} B_{n}\right) \approx 0$ or $\mathbf{u} B_{n} \approx \mathbf{v}_{\mathrm{LCT}} B_{n}+$ $\nabla_{t} \times(\Psi \mathbf{n})$ where $\Psi$ is an arbitrary function of $\mathbf{r}$. Therefore, but for the $\Psi$ ambiguity, it follows $\mathbf{v}_{\mathrm{LCT}} \approx \mathbf{u}$ at regions with $B_{n} \neq 0$. This means that at the presence of non-zero horizonal fields the velocity $\mathbf{v}_{\mathbf{L C T}}$ determined using the LCT method is contributed not only by the horizonal component of plasma motion, but also its vertical component. An important consequence is that the two terms in Eq (1) may be combined into one

$$
d H / d t=-2 \oint\left(\mathbf{u} \cdot \mathbf{A}_{p}\right) B_{n} d S \approx-2 \oint\left(\mathbf{v}_{\mathbf{L C T}} \cdot \mathbf{A}_{p}\right) B_{n} d S .
$$

This indicates that the LCT method gives a measure of the total amount of helicity change, namely the sum of both the shearing term and the advection term. This is the important finding of Demoulin \& Berger (2003).

\section{Results and Conclusion}

It is now obvious that the LCT method can be applied to active regions in the phase of flux emergence in which the advection term may be significant. As an illustration, we apply the LCT method to the active region NOAA 10365 that 

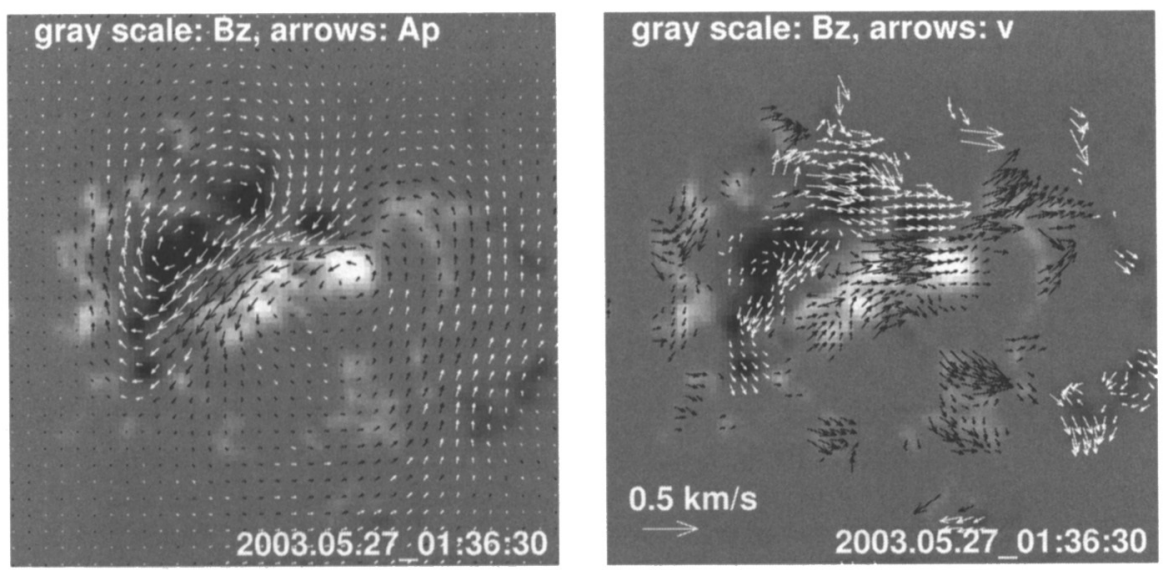

Figure 1. left: $\mathbf{A}_{\mathrm{p}}$ field (arrow) superposed on $B_{n}$ field (gray-sclae) in NOAA 10365. right: $\mathbf{v}_{\mathrm{LCT}}$ field (arrow) superposed on $B_{n}$ field (gray-scale).

was under emergence while it stayed near the disk center in 2003 May. It was the only dominant, flare-productive active region on the disk during the days of our interest. Here we present the results obtained from the 96 -min cadence full-disk magnetograms taken by SOHO/MDI.

Figure 1 shows the spatial distributions of $B_{n}, \mathbf{A}_{\mathrm{p}}$ and $\mathbf{v}_{\mathrm{LCT}}$ at an instant as determined using the method described by Chae (2001). It can be seen from the figure that the vector potential $\mathbf{A}_{\mathrm{p}}$ is the strongest on the polarity inversion line, and strong shearing motion occurs near the polarity inversion line, which should be important in the transfer of magnetic helicity.

Figure 2 shows that the magnetic fluxes of the active region steadily increased for several days from its birth. The total fluxes that newly emerged are found to be about $1.4 \times 10^{22} \mathrm{Mx}$ (positive flux) and $1.1 \times 10^{22} \mathrm{Mx}$ (negative flux). The right panel of the figure shows that magnetic helicity was transferred through the photosphere at a rate of up to $2.5 \times 10^{41} \mathrm{Mx}^{2} \mathrm{~h}^{-1}$. Figure 2 also shows the accumulated amount of helicity as a function of time. It is obvious from the figure that the helicity steadily increased as the fluxes did, but not in proportion to the fluxes. The final amount of magnetic helicity that was transferred during this emergence period is found to be $8 \times 10^{42} \mathrm{Mx}^{2}$, which yields $n \equiv H / \Phi^{2} \approx 0.05$ for an estimate of number of twist of the active region as a whole.

We have examined the dependence of the LCT method on the two free LCT parameters - apodizing window size and time interval - by comparing results from high cadence, high resolution magnetograms with those from 96-minute cadence, full-disk magnetograms. As a result we have found that smaller values of these parameters usually result in larger-amplitude temporal fluctuations of the rate of helicity. Its average taken over the time interval of about an hour or longer, however, is found to be very insensitive to the two parameters. This may be because short-lived, small-scale flows is insignificant in transferring magnetic helicity. This result supports that the LCT method may be safely applied to 

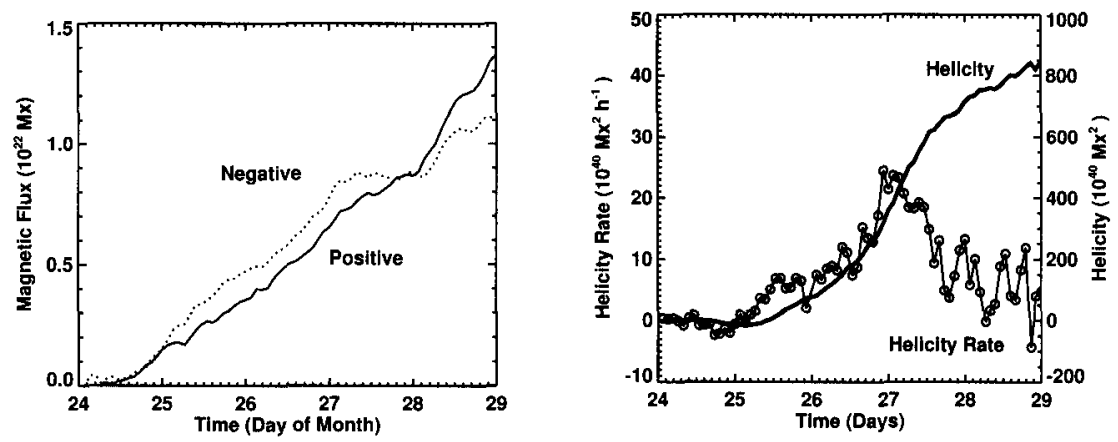

Figure 2. The temporal variations of positive and negative fluxes (left), and the rate and accumulated amount of magnetic helicity change (right) in NOAA 10365 observed in May 2003.

96-minute cadence full-disk MDI magnetograms as far as the magnetic helicity budget of active regions is concerned.

It is concluded that the LCT method is a practical and effective tool to infer the rate of helicity change in active regions. Its application to different active regions of comparable size by different authors (Chae et al. 2001; Nindos \& Zhang 2002; Nindos et al. 2003; the present study) has revealed that significant injection of magnetic helicity may occur both during and after flux emergence with a time scale of a few days, suggesting that typical helicity budget of major active regions with flux $10^{22} \mathrm{Mx}$ may be equal to or less than $\sim 10^{43} \mathrm{Mx}^{2}$ or twisted at most 0.1 turns as a whole.

Acknowledgments. This work was supported by the BK21 Project, and the KOSEF grant R14-2002-043-0100-0, and the National Research Laboratory M10104000059-01J00002500 of the Korean government.

\section{References}

Berger, M. A., \& Field, G. B. 1984, J. Fluid Mech., 147, 133

Chae, J. 2001, ApJ, 560, L95

Chae, J., Wang, H., Qiu, J., Goode, P. R., Strous, L., \& Yun, H. S. 2001, ApJ, 560, 476

Demoulin, P. \& Berger, M. A. 2003, Solar Physics, 215, 203

Kusano, K., Maeshiro, T., Yokoyama, T., \& Sakurai, T. 2002, ApJ, 577, 501

Moon, Y.-J., Chae, J., Choe, G. S., Wang, H., Park, Y. D., Yun, H. S., Yurchyshyn, V., \& Goode, P. R. 2002a, ApJ, 574, 1066

Moon, Y.-J., Chae, J., Wang, H., Choe, G. S., \& Park, Y. D. 2002b, ApJ, 580, 528

Nindos, A. \& Zhang, H. 2002, ApJ, 573, L133

Nindos, A., Zhang, J., \& Zhang, H. 2003, ApJ, 594, 1033 